\title{
Synchronous double primary cancer - intrahepatic cholangiocarcinoma with bone metastases and thyroid carcinoma: A case report
}

\author{
QING-LIANG WANG ${ }^{1}$, XIAO-JIE LI ${ }^{2}$, KUN ZHAO $^{1}$, BO LIU $^{1}$ and XIAO-MING YE ${ }^{1}$ \\ Departments of ${ }^{1}$ General Surgery and ${ }^{2}$ Laboratory Medicine, Ling Nan Hospital, \\ The Third Affiliated Hospital of Sun Yat-Sen University, Guangzhou, Guangdong 510530, P.R. China
}

Received November 22, 2014; Accepted September 28, 2015

DOI: $10.3892 / \mathrm{ol} .2015 .3822$

\begin{abstract}
There is a low incidence of multiple primary cancer, particularly when the cancer is synchronous. The present report presents a case of synchronous double primary malignancies. A 58-year-old woman was admitted to Ling Nan Hospital (Guangzhou, China) complaining of pain in the left hip. $\mathrm{X}$-ray revealed an osteolytic lesion and further examination indicated the presence of double primary cancer, consisting of hepatic cholangiocarcinoma and thyroid carcinoma. Biopsy of the osteolytic lesion showed a metastatic adenocarcinoma of unknown origin. Subsequently, final diagnosis was confirmed by I-131 scan and liver lesion biopsy. The patient received positive multidisciplinary treatments and survived for 9 months following diagnosis. The results of the present case suggest that multiplicity of primary malignancy is not necessarily an indicator of poor prognosis, as long as effective diagnosis and adequate disease management are achieved.
\end{abstract}

\section{Introduction}

Despite being first reported by Billroth in 1879 (1), until now, the occurrence of multiple primary malignancies in a single patient has been relatively rare. Simultaneous detection of malignancies in the thyroid and liver represents an uncommon event, despite the increasing overall incidence of malignancies. Intrahepatic cholangiocarcinoma (ICC) is a rare histopathological type of primary liver cancer, which accounts for only $5-10 \%$ of liver cancer (2). Common metastatic sites of ICC are regional lymph nodes and adjacent organs, while distant bone metastasis is rare (3). The prognosis of ICC remains poor due

Correspondence to: Dr Xiao-Ming Ye, Department of General Surgery, Ling Nan Hospital, The Third Affiliated Hospital of Sun Yat-Sen University, 2693 Kai Chuang Avenue, Guangzhou, Guangdong 510530, P.R. China

E-mail: james007y@sina.cn

Key words: double primary cancers, therapy, thyroid carcinoma, intrahepatic cholangiocarcinoma, bone metastases to its late clinical presentation and rapid recurrence, as well as the lack of adequate treatment strategies. In addition to curative resection, the potential benefit of other treatments in patient survival remains controversial (4). The present study reports a case of double primary cancer, comprising ICC with bone metastases in atypical locations, as well as thyroid carcinoma. The report details the therapeutic strategy used for inoperable and bone metastatic ICC, to provide useful information on this rare condition. Written informed consent was obtained from the patient's family.

\section{Case report}

A 58-year-old woman was admitted to Ling Nan Hospital (Guangzhou, China) in January 2010 complaining of repeated pain at the left hip for 2 months, accompanied by difficulty walking for 1 week. Clinical examination revealed a non-tender, palpable thyroid lump and abdominal mass at the epigastric area, while a gynecological examination was normal. The Patrick test, which is used to evaluate the pathology of the hip or sacroiliac joint, was performed by flexing, abducting and externally rotating each leg in turn. If pain is elicited on the ipsilateral side anteriorly, it is suggestive of a hip joint disorder on the same side. If pain is elicited on the contralateral side posteriorly around the sacroiliac joint, it is suggestive of pain mediated by dysfunction in that joint. In the current patient, the test was positive on the left, but negative on the right.

Pelvic X-ray revealed an osteolytic lesion at the upper margin of the left acetabulum (Fig. 1A). Ultrasound showed that the thyroid lump was a carcinoma located at the right lobe, and that there were multiple occupying lesions in the liver. Biopsy specimens of the osteolytic lesion identified a metastatic, moderately-differentiated adenocarcinoma of unknown origin. Bone radionuclide scanning and an I-131 total body scan were conducted to reach the final diagnosis (Fig. 2). Subsequently, ultrasound-guided fine-needle aspiration and core biopsy were performed. The smears revealed crowded clusters and sheets of malignant cells with glandular arrangement, while immunohistochemistry revealed that the tumor cells were positive for cytokeratin (CK) 7 and CK19, but negative for CK20. Therefore, ICC with bone metastases was diagnosed. 

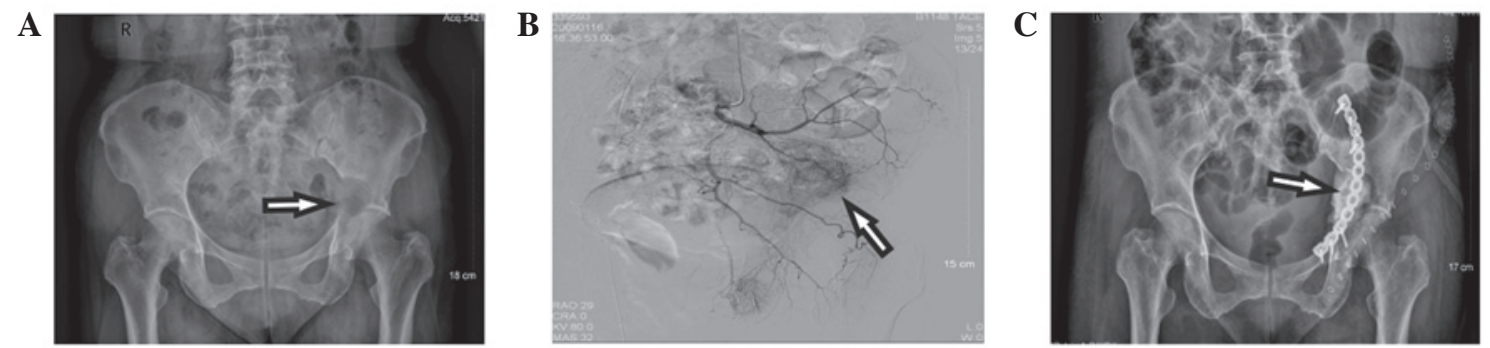

Figure 1. Anteroposterior radiograph of the pelvis. (A) X-ray revealed an osteolytic lesion (arrow). (B) Arteriography and embolization for the metastatic bone tumor (arrow). (C) Follow-up X-ray following metastatic tumor curettage, cement infilling and internal plate fixation (arrow).
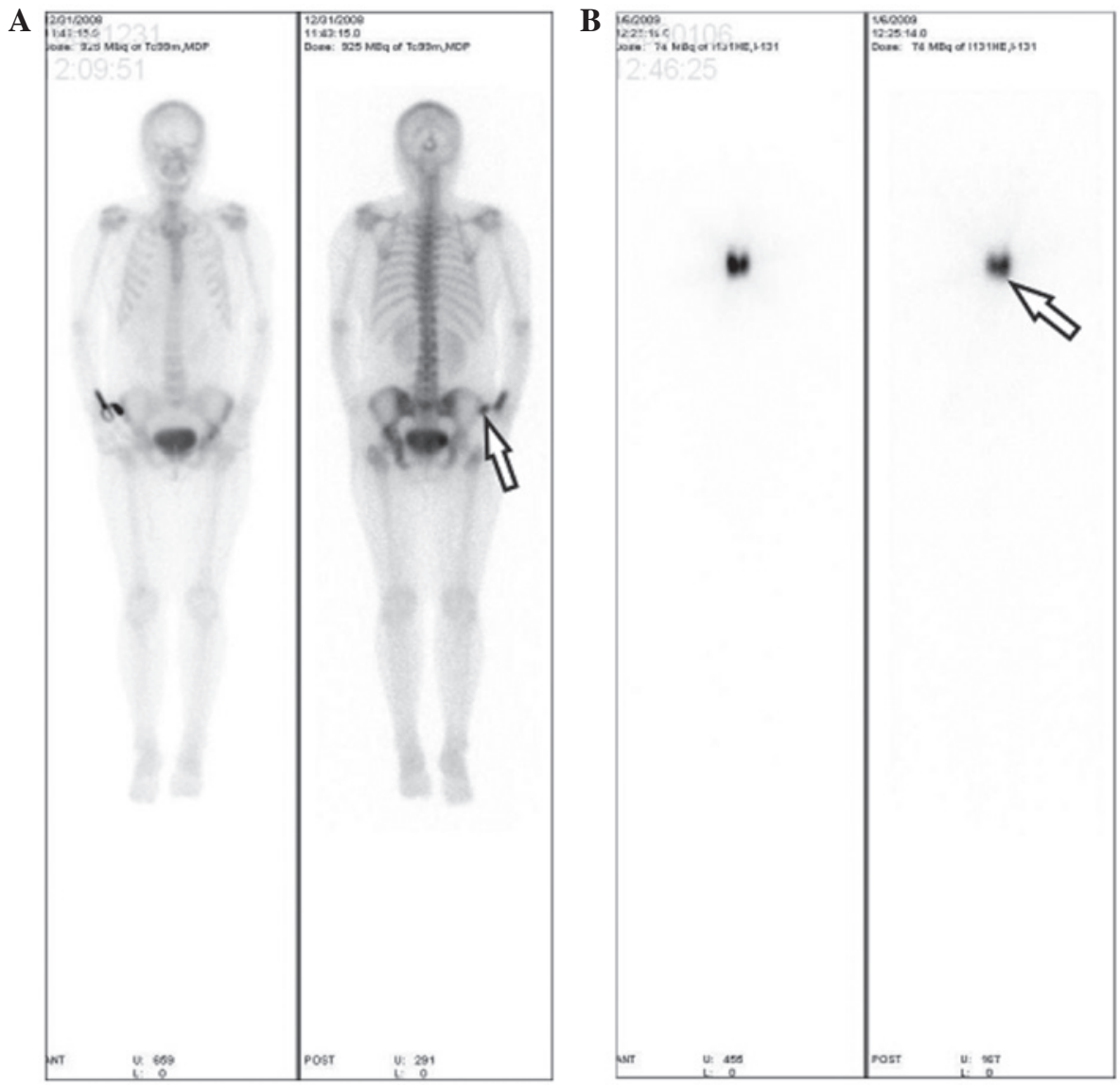

Figure 2. (A) Whole body bone scintigraphy revealed increased uptake in the left acetabulum (arrow). (B) I-131 whole body scan revealed there was no tissue exhibiting abnormal uptake, except the thyroid (arrow).

Transarterial chemoembolization (TACE) was used to control the primary lesion, while simultaneously, internal iliac arteriography and embolization were performed to control the metastatic bone tumor (Fig. 1B). One month later, the patient still experienced difficulty walking. Therefore, an operation comprising metastatic tumor curettage, cement infilling and internal plate fixation was performed (Fig. 1C). Several days subsequent to surgery, the patient was able to walk unaided, and the pain in the left hip was alleviated. Due to the poor results of the TACE, percutaneous port catheter system implantation via the left subclavian artery was used for further treatment. The patient subsequently commenced three courses of gemcitabine-based chemotherapy. Aggressive radiotherapy or single-fraction irradiation were not utilized for further treatment, at the request of the patient's family. The patient succumbed 9 months following initial diagnosis.

\section{Discussion}

Multiple primary malignancies are divided into two categories, synchronous and metachronous malignancies, based on the length of time between tumor diagnoses (5). Synchronous cancers are defined as those where secondary tumors occur simultaneously or within 6 months of diagnosis of the primary malignancy (6). Due to the increased life expectancy and improved screening programs, the early detection ratio for multiple primary malignancies is expected to increase (7). Among those patients with synchronous multiple malignancies, double primary cancer was most commonly observed, 
while cases of triple cancer or more occurred in $<1 \%$ of patients (8).

ICC, arising from the epithelial cells of the intrahepatic biliary tree, is a rare type of primary liver cancer, which has exhibited a global increasing trend in recent years (9). The incidence of ICC increases with age, and peaks in the sixth to seventh decade of life (4). To date, ICC synchronous with HCC (10), ampullary carcinoid (11), lymphoma (12), lung squamous cell carcinoma (13, lymphoepithelioma (14) and renal cell carcinoma (15) have been reported, while few cases have been reported synchronous with thyroid carcinoma. ICC typically metastasizes to the regional lymph nodes, liver, lungs and adjacent organs. Bone metastasis from ICC to the acetabular is particularly rare. By contrast, bone metastases are more commonly associated with other primary tumor locations, for example the lung, breast and thyroid. Therefore, the metastatic osteolytic adenocarcinoma of unknown origin was more likely to have developed from the thyroid carcinoma than the ICC. Unexpectedly, the metastatic lesion identified in the present case was confirmed to have arisen from ICC, as indicated by I-131 scan and liver lesion biopsy.

Although the mechanisms underlying the occurrence of multiple primary cancer are yet to be fully elucidated, there are a number of established risk factors for ICC. To the best of our knowledge, several factors have previously been defined, including parasitic infection, primary sclerosing cholangitis, biliary-duct cysts and hepatolithiasis (16). In addition, other potential risk factors include hepatitis, cirrhosis, diabetes, inflammatory bowel disease, alcohol drinking and tobacco smoking (17). In terms of the present case, diabetes and chronic hepatitis $\mathrm{B}$ virus infection may be responsible for the occurrence of ICC.

Currently, there is no established therapy for the treatment of synchronous primary cancer following diagnosis. Treatment may involve curative surgical resection of each cancer, radiotherapy and chemotherapy, depending on the location of the tumors. In the present case of synchronous primary cancer, due to the advanced disease stage, the aims of palliative therapy were to control local tumor growth and relieve symptoms, while improving and preserving the patient's quality of life.

Previously published data have reported that there are four categories of metastatic acetabular tumor (18). The majority of cases were detected by painful symptoms or neurological signs resulting from structural damage, periosteal irritation and nerve entrapment (19). For these patients, resection and reconstruction of the metastatic lesions was able to improve quality of life by increasing mobility and reducing pain (20). In the present case, minimally invasive treatment failed to control the metastatic tumor, therefore surgical intervention was utilized and achieved effective results.

Preoperative TACE has been used to control primary liver neoplasm (21). However, the outcome for certain patients is unsatisfactory due to the malignant nature of the tumor. ICC has been shown to be resistant to common chemotherapy, with an unacceptably low response rate (22). Various chemotherapeutic agents have been evaluated for the treatment of ICC in numerous clinical trials, however no standard chemotherapeutic regimen has yet been identified (23-25). Double gemcitabine and cisplatin therapy is currently considered the first-line therapy for patients with advanced disease (26).
Although no randomized trials have been conducted to support the use of adjuvant treatment, gemcitabine-based chemotherapy combined with TACE may show promising efficacy with respect to survival and quality of life for advanced ICC with bone metastasis. Patients with poor prognosis may potentially benefit from the shorter treatment duration, due to their limited life expectancy.

Despite their infrequency, double primary malignancies, for example intrahepatic cholangiocarcinoma with bone metastases and thyroid carcinoma, should be considered seriously. Multiplicity of primary malignancies itself is not necessarily indicative of a poor prognosis. The results of the present case suggest that early diagnosis, together with positive multidisciplinary treatments, may show promising efficacy with respect to patient survival and quality of life. A study of patients from a large, multicenter and multigeographical cohort may facilitate validation of these results and reach more powerful conclusions.

\section{References}

1. Billroth $\mathrm{T}$ and Chirurgische Klinik Wien: Nebst einem Gesammt-Bericht über die chirurgischen Kliniken in Zürich und Wien während der Jahre 1860-1876: Erfahrungen auf dem Gebiet der practischen Chirurgie. Hirschwald Berlin, 1879 (In German).

2. Tsai S, Nathan H and Pawlik TM: Primary liver cancer: Intrahepatic cholangiocarcinoma emerges from the shadows. Updates Surg 62: 5-9, 2010.

3. Shirabe K, Shimada M, Harimoto N, Sugimachi K, Yamashita Y, Tsujita E and Aishima S: Intrahepatic cholangiocarcinoma: Its mode of spreading and therapeutic modalities. Surgery 131: S159-S164, 2002.

4. Yang J and Yan LN: Current status of intrahepatic cholangiocarcinoma. World J Gastroenterol 14: 6289-6297, 2008.

5. Suzuki T, Takahashi H, Yao K, Inagi K, Nakayama M, Makoshi T, Nagai $\mathrm{H}$ and Okamoto M: Multiple primary malignancies in the head and neck: A clinical review of 121 patients. Acta Otolaryngol Suppl 547: 88-92, 2002.

6. Moertel CG: Multiple primary malignant neoplasms: Historical perspectives. Cancer 40 (Suppl): 1786-1792, 1977.

7. Moertel CG: Incidence and significance of multiple primary malignant neoplasms. Ann NY Acad Sci 114: 2012.

8. Luciani A and Balducci L: Multiple primary malignancies. Semin Oncol 31: 264-273, 2004.

9. Poultsides GA, Zhu AX, Choti MA and Pawlik TM: Intrahepatic cholangiocarcinoma. Surg Clin North Am 90: 817-837, 2010.

10. Jung KS, Chun KH, Choi GH, Jeon HM, Shin HS, Park YN and Park JY: Synchronous development of intrahepatic cholangiocarcinoma and hepatocellular carcinoma in different sites of the liver with chronic B-viral hepatitis: Two case reports. BMC Res Notes 6: 520, 2013

11. Takeuchi Y, Nagata K, Yokota T, Handa M, Matsunaga H, Nishio Y and Kusugami K: Von Recklinghausen disease associated with intrahepatic cholangiocarcinoma and ampullary carcinoid. Nihon Naika Gakkai Zasshi 90: 2467-2469, 2001 (In Japanese).

12. Fwu CW, Chien YC, You SL, Nelson KE, Kirk GD, Kuo HS, Feinleib M and Chen CJ: Hepatitis B virus infection and risk of intrahepatic cholangiocarcinoma and non-Hodgkin lymphoma: A cohort study of parous women in Taiwan. Hepatology 53: 1217-1225, 2011.

13. Murakami T, Nishikawa H, Koshikawa Y, Okabe Y, Wakasa T and Osaki Y: Double primary cancers: Intrahepatic cholangiocarcinoma with myocardial metastases and lung squamous cell carcinoma. Intern Med 51: 2329-2335, 2012.

14. Henderson-Jackson E, Nasir NA, Hakam A, Nasir A and Coppola D: Primary mixed lymphoepithelioma-like carcinoma and intra-hepatic cholangiocarcinoma: A case report and review of literature. Int J Clin Exp Pathol 3: 736-741, 2010.

15. Anthony MP, Mak H and Khong PL: An unusual case of synchronous renal cell carcinoma in a horseshoe kidney and intrahepatic cholangiocarcinoma. Clin Nucl Med 34: 922-923, 2009. 
16. Palmer WC and Patel T: Are common factors involved in the pathogenesis of primary liver cancers? A meta-analysis of risk factors for intrahepatic cholangiocarcinoma. J Hepatol 57: 69-76, 2012.

17. Tyson GL and El-Serag HB: Risk factors for cholangiocarcinoma. Hepatology 54: 173-184, 2011.

18. Harrington KD: The management of acetabular insufficiency secondary to metastatic malignant disease. J Bone Joint Surg Am 63: 653-664, 1981.

19. Shahid M, Saunders T, Jeys L and Grimer R: The outcome of surgical treatment for peri-acetabular metastases. Bone Joint J 96-B: 132-136, 2014.

20. Hoell S, Dedy N, Gosheger G, Dieckmann R, Daniilidis K and Hardes J: The Burch-Schneider cage for reconstruction after metastatic destruction of the acetabulum: Outcome and complications. Arch Orthop Trauma Surg 132: 405-410, 2012.

21. Bester L, Meteling B, Boshell D, Chua TC and Morris DL: Transarterial chemoembolisation and radioembolisation for the treatment of primary liver cancer and secondary liver cancer: a review of the literature. J Med Imaging Radiat Oncol 58: 341-352, 2014.
22. Furuse J, Kasuga A, Takasu A, Kitamura H and Nagashima F: Role of chemotherapy in treatments for biliary tract cancer. J Hepatobiliary Pancreat Sci 19: 337-341, 2012.

23. Valle J, Wasan H, Palmer DH, Cunningham D, Anthoney A, Maraveyas A, Madhusudan S, Iveson T, Hughes S, Pereira SP,et al; ABC-02 Trial Investigators: Cisplatin plus gemcitabine versus gemcitabine for biliary tract cancer. N Engl J Med 362: 1273-1281, 2010.

24. Gruenberger B, Schueller J and Heubrandtner U, Wrba F, Tamandl D, Kaczirek K, Roka R, Freimann-Pircher S and Gruenberger T: Cetuximab, gemcitabine, and oxaliplatin in patients with unresectable advanced or metastatic biliary tract cancer: A phase 2 study. Lancet Oncol 11: 1142-1148, 2010.

25. Eckmann KR, Patel DK, Landgraf A, Slade JH, Lin E, Kaur H, Loyer E, Weatherly JM and Javle M: Chemotherapy outcomes for the treatment of unresectable intrahepatic and hilar cholangiocarcinoma: A retrospective analysis. Gastrointest Cancer Res 4: 155-160, 2011.

26. Maithel SK, Gamblin TC, Kamel I, Corona-Villalobos CP, Thomas M and Pawlik TM: Multidisciplinary approaches to intrahepatic cholangiocarcinoma. Cancer 119: 3929-3942, 2013. 